\title{
Characterization and Modeling of the Liquid Phase of Deep Eutectic Solvents Based on Fatty Acids/Alcohols and Choline Chloride
}

\author{
Emanuel A. Crespo, ${ }^{\dagger}+\subset$ Liliana P. Silva, ${ }^{\dagger}$ Mónia A. R. Martins, ${ }^{\dagger}$ Luis Fernandez, ${ }^{\S}$ Juan Ortega, ${ }^{\S}$

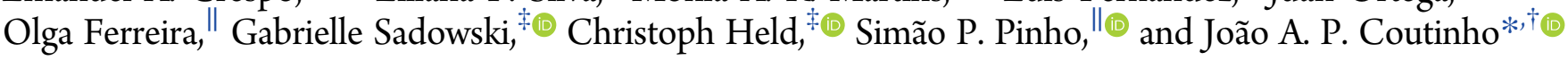 \\ ${ }^{\dagger}$ CICECO - Aveiro Institute of Materials, Department of Chemistry, University of Aveiro, 3810-193 Aveiro, Portugal \\ ${ }^{\ddagger}$ Laboratory of Thermodynamics, Department of Biochemical and Chemical Engineering, TU Dortmund, 44227 Dortmund, \\ Germany \\ ${ }^{\S}$ Laboratorio de Termodinamica y Fisicoquímica de Fluidos, 35071 - Parque Científico-Tecnológico, Universidad de Las Palmas de \\ Gran Canaria, Canary Islands, Spain \\ "Associate Laboratory LSRE-LCM, Departamento de Tecnologia Química e Biológica, Instituto Politécnico de Bragança, 5301-857 \\ Bragança, Portugal
}

\section{Supporting Information}

\begin{abstract}
The solid-liquid equilibria phase diagrams of eight eutectic systems formed by choline chloride and fatty acids, or fatty alcohols, were measured to characterize the nonideality of the liquid phase of these systems, commonly reported in the literature as examples of type III deep eutectic solvents (DESs), and to evaluate the best modeling approaches to their description. Most of these systems are shown to present only slight deviations from ideal behavior, resulting from a fine balance of the hydrogen bonding between the hydroxyl/carboxylic groups with the chloride anion and the interactions present in the pure compounds. The phase diagrams measured were modeled with an associative equation of state $(\mathrm{EoS})$ and a $g^{\mathrm{E}}$ model. As an EoS, the perturbed-chain statistical associating fluid theory (PC-SAFT) was used, and this model was able to accurately describe the experimental data and to provide reliable estimates of the eutectic points using just a single binary temperature-dependent interaction parameter that often correlates with the acid/alcohol chain length. The performance of PC-SAFT was further compared with the $g^{\mathrm{E}}$ model, a non-random two-liquid model (NRTL), and was found to provide a better description of the experimental data, especially for the more nonideal systems. Ultimately, the data gathered, and the molecular modeling, allowed the discussion of the behavior of fatty acids or fatty alcohols as hydrogen bond donors in choline chloride-based DESs.
\end{abstract}

\section{INTRODUCTION}

Deep eutectic solvents (DESs) exhibit a wide range of properties which make them an attractive family of solvents for different applications in catalysis, organic synthesis, dissolution and extraction processes, electrochemistry, and material chemistry. ${ }^{1,2}$ The most common components used in DES formulations are quaternary ammonium salts, particularly choline chloride $([\mathrm{Ch}] \mathrm{Cl})$, because of its low toxicity, biodegradability, and economic synthesis, combined with urea, $^{3,4}$ carboxylic acids, ${ }^{5-7}$ alcohols or polyols, ${ }^{8-11}$ amino acids, ${ }^{12}$ or sugars. ${ }^{13,14}$

Even though a large number of DESs have been reported in the literature, the actual number of solid-liquid phase diagrams investigated is surprisingly small despite the important information they can provide on the donor-acceptor interactions and the range of compositions and temperatures for using these solvents in processes. ${ }^{3,5,12}$ This limits the development of models to describe the properties and behavior of these solvents, in particular their liquid-phase nonideality and thus the ability to design new DESs using computer-aided molecular design approaches instead of the trial and error currently used. Additionally, in order to screen solvents with particular characteristics and specific ranges of eutectic temperatures, knowledge of the relation between the structural characteristics of the compounds and the eutectic points is necessary. Thus, studies of solid-liquid phase diagrams focused on comparable systems (same functional groups and different chain lengths, same chain lengths and different functional groups, etc.) are required. Moreover, although carboxylic acids and alcohols are commonly reported as possible hydrogen bond donors (HBDs) for the formation of type III DESs, ${ }^{5-11}$ the liquid-phase nonideality of fatty acids/alcohols: $[\mathrm{Ch}] \mathrm{Cl}$ based DESs was never investigated.

Received: June 9, 2017

Revised: September 17, 2017

Accepted: September 26, 2017

Published: September 26, 2017 
Table 1. Source, Purity, and Melting Properties of the Chemicals Used in This Work

\begin{tabular}{llcccc} 
& & & \multicolumn{2}{c}{$T_{\text {fus }}(\mathrm{K})$} & \multicolumn{1}{c}{$\Delta_{\text {fus }} H\left(\mathrm{~J} \cdot \mathrm{mol}^{-1}\right)$} \\
\cline { 3 - 5 } compound & \multicolumn{1}{c}{ source } & mass purity \% & - & $597^{17}$ & $4300^{17}$ \\
{$[\mathrm{Ch}] \mathrm{Cl}$} & Acros Organic & 98 & $311.7 \pm 0.3$ & $311.10^{20}$ & $45810^{20}$ \\
1-tetradecanol & Aldrich & 97 & $324.4 \pm 0.2$ & $322.90^{21}$ & $60960^{21}$ \\
1-hexadecanol & Aldrich & 99 & $332.9 \pm 0.4$ & $331.34^{21}$ & $65350^{21}$ \\
1-octadecanol & Aldrich & 99 & $304.8 \pm 0.2$ & $305.48^{20}$ & $27500^{7}$ \\
capric acid & Alfa Aesar & 99 & $317.5 \pm 0.3$ & $318.48^{20}$ & $34690^{7}$ \\
lauric acid & Acros Organics & 99 & $327.0 \pm 0.2$ & $328.93^{20}$ & $45750^{7}$ \\
myristic acid & Acros Organics & 99 & $336.8 \pm 0.2$ & $336.36^{21}$ & $51020^{7}$ \\
palmitic acid & Riedel de Haen & 98 & $343.7 \pm 0.3$ & $344.04^{21}$ & $61360^{7}$ \\
stearic acid & Acros Organics & 97 & & \\
\hline
\end{tabular}

In our previous work, ${ }^{7}$ the formation of DESs composed of symmetrical tetraalkylammonium chlorides and fatty acids was reported, but the different chemical structure of $[\mathrm{Ch}] \mathrm{Cl}$ and the presence of one additional hydroxyl group in the DES moiety can significantly influence the interactions between the DES constituents. Therefore, in this work, the solid-liquid phase diagrams of eight different eutectic mixtures formed by choline chloride and fatty alcohols or fatty acids are reported. The experimental data were measured using a visual detection technique, and their thermodynamic consistency was analyzed using tests recently proposed in the literature. ${ }^{15-17}$ The phase diagrams measured were then modeled using the perturbedchain statistical associating fluid theory (PC-SAFT) equation of state $(\mathrm{EoS})^{18}$ which is able to explicitly account for the association between the DES constituents. The performance of this advanced EoS was compared with non-random two-liquid model (NRTL), ${ }^{19}$ which is an activity coefficient model using a similar or higher number of binary parameters than usually applied in PC-SAFT. Finally, the modeling results of the solidliquid equilibrium of these systems were then used to assess the nonideality of the liquid phase that is paramount to understand the nature of a DES.

\section{MATERIALS AND METHODS}

2.1. Materials. Source and purity of the compounds used in this work are detailed in Table 1. Experimental melting temperatures for the fatty alcohols and fatty acids measured in this work are also compared with literature values in Table 1, showing a good agreement. For $[\mathrm{Ch}] \mathrm{Cl}$, the melting properties proposed in our previous work were used. ${ }^{17}[\mathrm{Ch}] \mathrm{Cl}$ was purified before use under vacuum (0.1 $\mathrm{Pa}$ and $298 \mathrm{~K})$ and constant stirring, for at least $72 \mathrm{~h}$. The water content was measured using a Metrohm 831 Karl Fischer coulometer, with the analyte Hydranal Coulomat AG from Riedel-de Haën, and was found to be below $700 \mathrm{ppm}$. Mixtures of choline chloride with 1-decanol and 1-dodecanol were also prepared; however, these mixtures separated into two liquid phases and thus were excluded from investigations within this work.

2.2. Methods. The solid-liquid phase diagrams were measured using a melting point device model M-565 by Bucchi $(100-240 \mathrm{~V}, 50-60 \mathrm{~Hz}, 150 \mathrm{~W})$ with a temperature resolution of $0.1 \mathrm{~K}$. A temperature gradient of 0.1 and $0.5 \mathrm{~K} \cdot \mathrm{min}^{-1}$ was used for pure components and binary mixtures, respectively. All samples were measured at least two times. Mixtures were prepared at room temperature inside a dry-argon glovebox, using an analytical balance model ALS 220-4N from Kern with an accuracy of $\pm 0.002 \mathrm{~g}$. Whenever possible mixtures were heated under stirring until complete melting and then recrystallized and mashed. The powder was filled into a glass capillary. The estimated reproducibility of the measurements is of $1.3 \mathrm{~K}$.

\section{THEORY}

3.1. PC-SAFT. Modeling the phase equilibrium and thermodynamic properties of complex systems like DESs, in which molecules exhibit associative interactions, is still a challenging task although needed in chemical industry and academia. Clearly the most apparent progress toward the development of EoSs with such capabilities is achieved through the application of statistical mechanics. EoSs based on statistical mechanics are able to explicitly account for the influence of different structural effects and interactions (e.g., anisotropic association and electrostatics) on the thermodynamic properties and phase behavior. A concept of such EoS was proposed by Chapman et al. ${ }^{22-25}$ based on Wertheim's first-order thermodynamic perturbation theory, ${ }^{26-29}$ statistical associating fluid theory (SAFT). In the framework of SAFT, molecules are modeled as associating chains formed of equally sized bonded spherical segments with short-range attractive sites. SAFT-type EoSs are generally written as a sum of different contributions to the residual Helmholtz energy, $A^{\text {res }}$, defined as the difference between the system's Helmholtz energy and that of an ideal gas at the same molar density and temperature:

$$
\begin{aligned}
& \frac{A^{\text {res }}}{N k_{\mathrm{B}} T}=\frac{A}{N k_{\mathrm{B}} T}-\frac{A^{\text {id }}}{N k_{\mathrm{B}} T} \\
& \frac{A^{\text {res }}}{N k_{\mathrm{B}} T}=\frac{A^{\text {mono }}}{N k_{\mathrm{B}} T}+\frac{A^{\text {chain }}}{N k_{\mathrm{B}} T}+\frac{A^{\text {assoc }}}{N k_{\mathrm{B}} T}
\end{aligned}
$$

Several modifications have been proposed to the original SAFT model which essentially correspond to different choices for the monomer fluid and different theoretical approaches for the calculation of its Helmholtz energy. PC-SAFT ${ }^{18}$ is among the most prominent modifications of SAFT and has already been applied to model DESs. ${ }^{7,30,31}$ PC-SAFT considers a hardchain, rather than a hard-sphere, as the reference system for the application of the perturbation theories to obtain the dispersion contribution; thus, the residual Helmholtz energy is written as

$$
\frac{A^{\text {res }}}{N k_{\mathrm{B}} T}=\frac{A^{\mathrm{hc}}}{N k_{\mathrm{B}} T}+\frac{A^{\mathrm{disp}}}{N k_{\mathrm{B}} T}+\frac{A^{\text {assoc }}}{N k_{\mathrm{B}} T}
$$

In eqs $1-3$, the superscripts refer to the residual, ideal, monomer, chain formation, associative interactions, hard chain fluid, and dispersive interactions terms (res, id, mono, chain, assoc, hc, and disp, respectively) while $N, k_{\mathrm{B}}$, and $T$ stand for 
Table 2. PC-SAFT Pure-Component Parameters Used in This Work Using the 2B Association Scheme According to Huang and Radosz ${ }^{39}$

\begin{tabular}{|c|c|c|c|c|c|c|}
\hline component & $M_{\mathrm{w}}(\mathrm{g} / \mathrm{mol})$ & $m_{i}^{\text {seg }}$ & $\sigma_{i}(\AA)$ & $u_{i}(\mathrm{~K})$ & $\varepsilon^{A i B i}(\mathrm{~K})$ & $\kappa^{A i B i}$ \\
\hline$[\mathrm{Ch}] \mathrm{Cl}^{31}$ & 139.62 & 13.02 & 2.368 & 228.07 & 8000 & 0.2 \\
\hline capric acid ${ }^{7}$ & 172.26 & 7.1472 & 3.3394 & 242.46 & 2263.0 & 0.02 \\
\hline lauric $\operatorname{acid}^{36}$ & 200.32 & 7.2547 & 3.5244 & 252.97 & 3047.5 & 0.00338 \\
\hline myristic acid ${ }^{36}$ & 228.37 & 7.4126 & 3.6719 & 256.48 & 2252.5 & 0.04399 \\
\hline palmitic acid ${ }^{7}$ & 256.42 & 7.5599 & 3.8092 & 267.52 & 2291.4 & 0.02 \\
\hline stearic acid ${ }^{7}$ & 284.48 & 7.6146 & 3.9536 & 275.20 & 2351.6 & 0.02 \\
\hline 1-tetradecanol ${ }^{36}$ & 214.39 & 5.5424 & 4.0630 & 275.42 & 3551.5 & 0.00076 \\
\hline 1-hexadecanol ${ }^{36}$ & 242.45 & 6.1909 & 4.0764 & 273.14 & 3650.5 & 0.000747 \\
\hline 1-octadecanol ${ }^{a}$ & 270.50 & 6.5794 & 4.1645 & 278.15 & 3555.2 & 0.00265 \\
\hline
\end{tabular}
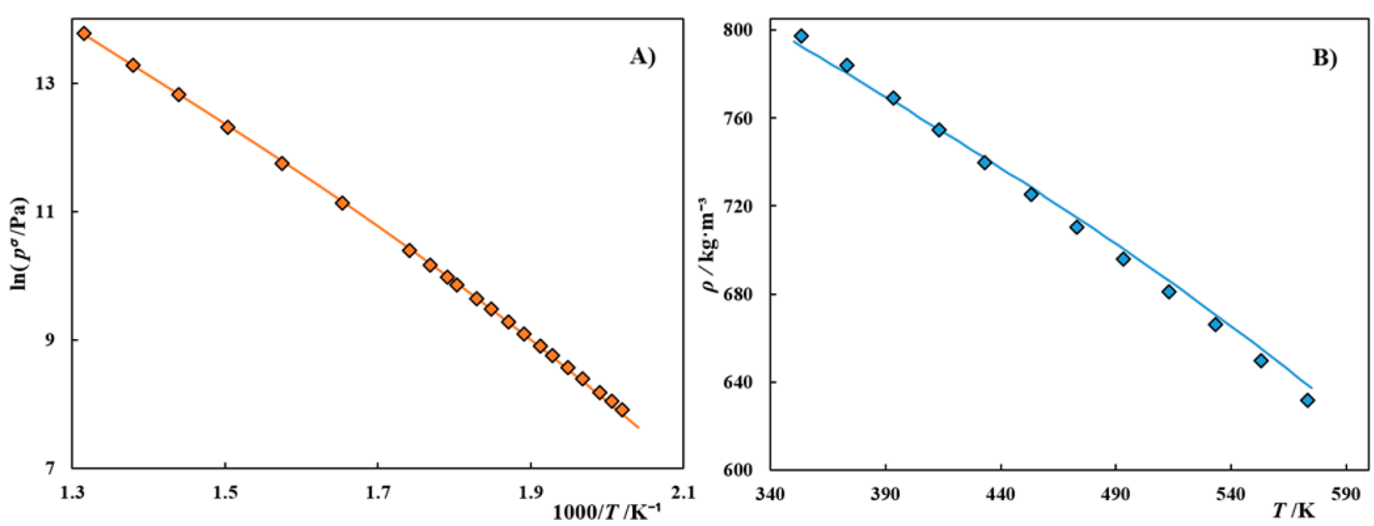

Figure 1. (A) Vapor pressures and (B) liquid densities for 1-octadecanol. The symbols represent experimental data taken from DIPPR database, ${ }^{37}$ while the solid lines depict the PC-SAFT modeling results using parameters from Table 2.

number of molecules, Boltzmann constant, and the system's absolute temperature, respectively.

In order to fully characterize an associating compound as those present in DESs, five pure-component PC-SAFT parameters are required: three nonassociating parameters (namely, the number of segments, $m_{i}^{\text {seg }}$; the segment diameter, $\sigma_{i}$; the dispersive energy between segments, $u_{i}$ ) and two association parameters (energy and bonding volume, $\varepsilon^{A i B i}$ and $\kappa^{A i B i}$, respectively). Moreover, an association scheme has to be specified for each associating component defining both the number and type of associative sites and the allowed interactions.

When dealing with mixtures the conventional LorentzBerthelot combining rules are used to determine the size and energy parameters between unlike segments. Here, one adjustable binary interaction parameter, $k_{i j}$, is introduced when needed to account for deviations of the cross-dispersion energy from its value obtained by the geometric mean.

$$
\begin{aligned}
& \sigma_{i j}=\frac{1}{2}\left(\sigma_{i}+\sigma_{j}\right) \\
& u_{i j}=\left(1-k_{i j}\right) \sqrt{u_{i} u_{j}}
\end{aligned}
$$

In this work, this was the only binary interaction parameter used to describe the phase diagrams of all the studied systems using a temperature-dependent expression for $k_{i j}$.

$$
k_{i j}=k_{i j_{-} \mathrm{a}}+(T / \mathrm{K}-298.15) \times k_{i j_{-} \mathrm{T}}
$$

Simple combining rules, proposed by Wolbach and Sandler, $^{32}$ are applied to obtain the cross-association parameters:

$$
\begin{aligned}
& \varepsilon^{A i B j}=\frac{1}{2}\left(\varepsilon^{A i B i}+\varepsilon^{A j B j}\right) \\
& \kappa^{A i B j}=\sqrt{\kappa^{A i B i} \cdot \kappa^{A j B j}}\left(\frac{\sqrt{\sigma_{i i} \cdot \sigma_{j j}}}{(1 / 2)\left(\sigma_{i i}+\sigma_{j j}\right)}\right)^{3}
\end{aligned}
$$

3.2. PC-SAFT Pure-Component Parameters. Several authors have reported the use of PC-SAFT to model IL solutions, ${ }^{31,33-35}$ where, to each IL molecule, two association sites are considered, which is referred to the $2 \mathrm{~B}$ association scheme mimicking the IL-cation and the IL-anion. Therefore, the molecular parameters for $[\mathrm{Ch}] \mathrm{Cl}$ were directly taken from Zubeir et al. ${ }^{31}$ and are reported in Table 2 .

Fatty acids and fatty alcohols have extensively been studied in the literature, ${ }^{36}$ and PC-SAFT pure-component parameters for them are available except for 1-octadecanol. For the latter, PCSAFT parameters were regressed in the present work from pure-fluid liquid densities and vapor pressures in a wide temperature range. By keeping the same association scheme (2B) applied by Albers et al. ${ }^{36}$ and Pontes et al. ${ }^{7}$ for fatty acids and fatty alcohols, PC-SAFT was able to accurately describe the experimental data. This is depicted in Figure 1, showing similar average absolute relative deviations (\%AARD as defined in eq 9, where exp and calc superscripts denote experimental and calculated values, respectively; $X$ stands for the thermodynamic property evaluated, and $N_{\exp }$ is the total number of experimental data points) to those reported in the literature for other fatty alcohols (\%AARD of $2.55 \%$ and $0.56 \%$ for vapor pressures and liquid densities, respectively). The purecomponent parameters used for the different fatty acids and alcohols studied in this work are presented in Table 2. 

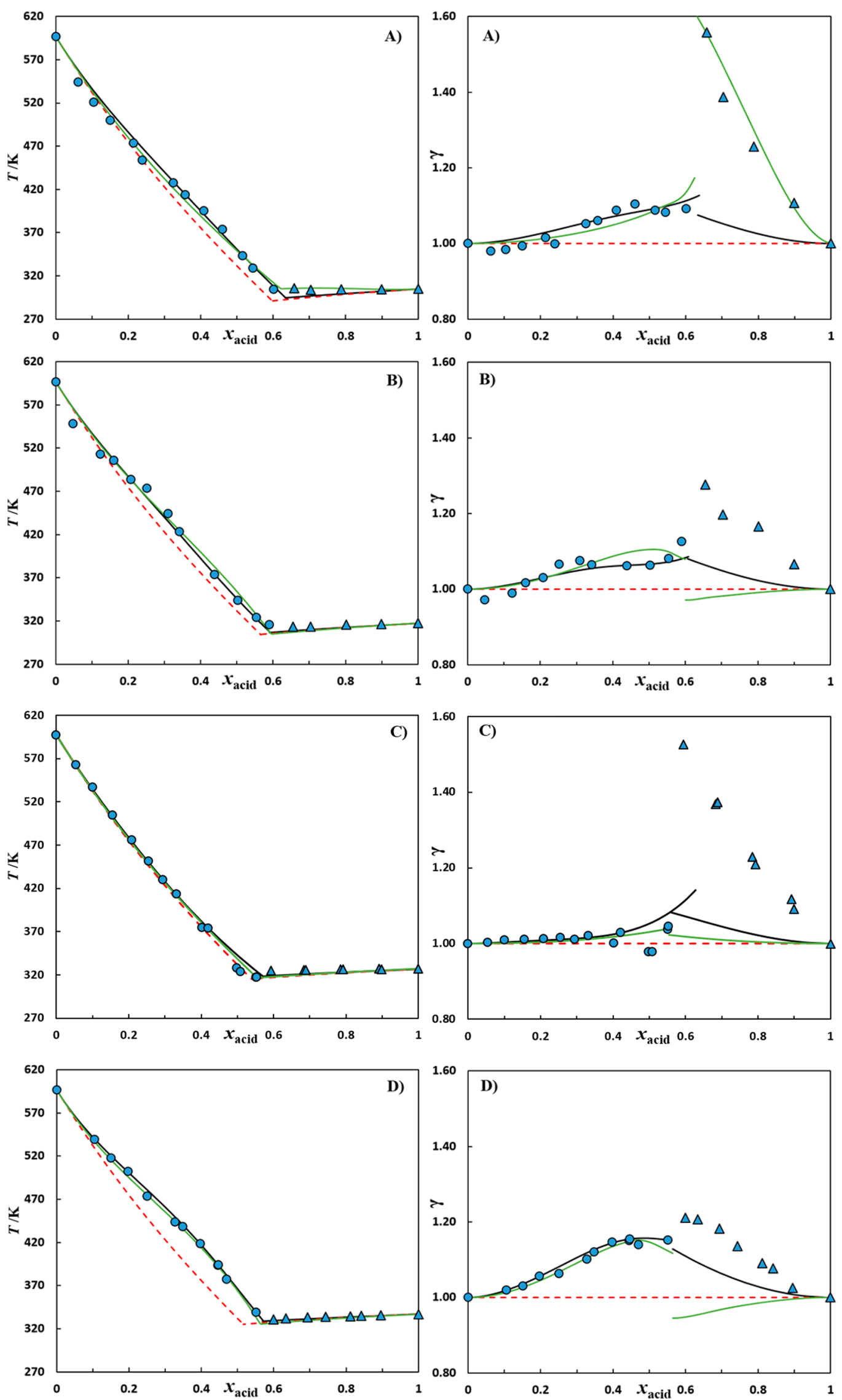

Figure 2. continued 

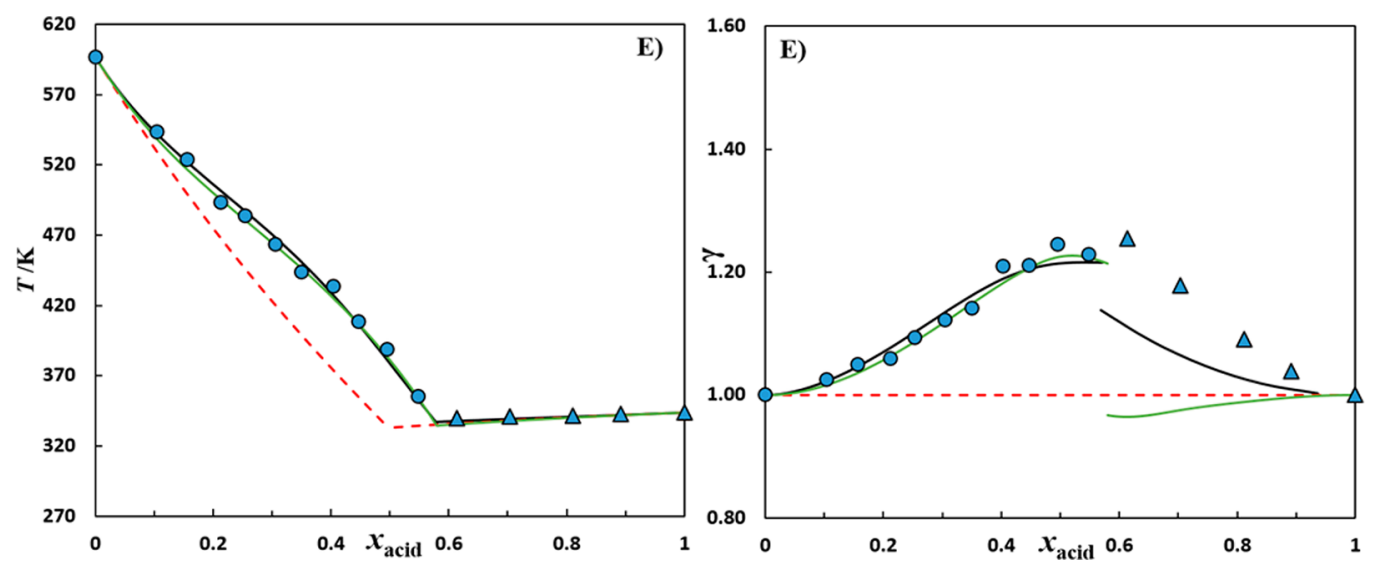

Figure 2. Solid-liquid phase diagrams for the binary mixtures composed of [Ch]Cl and (A) capric acid, (B) lauric acid, (C) myristic acid, (D) palmitic acid, (E) stearic acid. $\boldsymbol{\Delta}$ and $\bullet$ represent the fatty acid and $[\mathrm{Ch}] \mathrm{Cl}$ experimental solubility curves measured in this work, while the lines depict the modeling results, namely, red dashed line, ideal; solid black line, PC-SAFT; and solid green line, NRTL.

$$
\% \text { AARD }=\frac{1}{N_{\exp }} \sum_{i=1}^{N_{\text {exp }}} \frac{\left|X_{i}^{\text {calc }}-X_{i}^{\exp }\right|}{X_{i}^{\exp }} \times 100
$$

The consistency of the parameters proposed for 1 octadecanol was analyzed through the correlation of the nonassociating parameters with the molecular weight as typically performed for a homologous series, ${ }^{36,38}$ and coefficients of determination $\left(R^{2}\right)$ very close to unity were obtained:

$$
\begin{aligned}
& m_{i}^{\text {seg }}=0.01632 M_{\mathrm{w}}+2.164 ; \quad R^{2}=0.9898 \\
& m_{i}^{\text {seg }} \sigma_{i}^{3}\left(\AA^{3}\right)=1.8085 M_{\mathrm{w}}-16.600 ; \quad R^{2}=0.9988 \\
& m_{i}^{\text {seg }} u_{i}(K)=4.8881 M_{\mathrm{w}}+502.48 ; \quad R^{2}=0.9948
\end{aligned}
$$

3.3. Non-random Two-Liquid Model. Proposed by Renon and Prausnitz, NRTL ${ }^{19}$ is a local composition activity coefficient model. Its parameters are "energy differences" (between cross and self-interactions) and an additional parameter, $\alpha_{i j}$, the so-called nonrandomness factor, that is often treated as an additional adjustable parameter. NRTL has been applied with success to correlate solid-liquid equilibrium (SLE), vapor-liquid equilibrium (VLE), or liquid-liquid equilibrium (LLE) experimental data of a vast variety of systems, and although for moderately nonideal systems it offers no advantages over the simpler Margules or van Laar equations, for strongly nonideal mixtures, NRTL often gives a better representation of experimental data. Moreover, many works have been published where the NRTL equation was satisfactorily applied to correlate the LLE of systems containing DESs. ${ }^{40-45}$

In this work, the NRTL equation is used in its simplest form (without temperature dependency) to correlate the phase diagrams measured, and the expression for the excess Gibbs energy of a binary mixture is given by eq 13 with the activity coefficients given by eq 14 .

$$
\frac{g^{\mathrm{E}}}{R T}=x_{1} x_{2}\left(\frac{\tau_{21} G_{21}}{x_{1}+x_{2} G_{21}}+\frac{\tau_{12} G_{12}}{x_{2}+x_{1} G_{12}}\right)
$$

$$
\begin{gathered}
\ln \gamma_{i}=x_{j}^{2}\left[\tau_{j i}\left(\frac{G_{j i}}{x_{i}+x_{j} G_{j i}}\right)^{2}+\tau_{i j} \frac{G_{i j}}{\left(x_{j}+x_{i} G_{i j}\right)^{2}}\right] ; \\
i, j=\{1,2\} ; i \neq j \\
G_{i j}=\exp \left(-\alpha_{i j} \tau_{i j}\right) \text { with } \tau_{i j}=\frac{a_{i j}}{R T}
\end{gathered}
$$

3.4. Solid-Liquid Equilibria. The solubility of a single solute $i\left(x_{i}\right)$ in a liquid solvent can be described by the following simplified equation:

$$
\ln \left(x_{i} \gamma_{i}^{1}\right)=\frac{\Delta_{\text {fus }} H_{i}}{R}\left(\frac{1}{T_{\mathrm{m}, i}}-\frac{1}{T}\right)-\frac{\Delta_{\text {fus }} C p_{i}}{R}\left(\frac{T_{\mathrm{m}, i}}{T}-\ln \frac{T_{\mathrm{m}, i}}{T}-1\right)
$$

where $\gamma_{i}^{1}$ is the activity coefficient of component $i$ in the liquid phase (in this work calculated either with PC-SAFT or NRTL), $T$ the system absolute temperature, $R$ the universal gas constant $\left(R=8.314 \mathrm{~J} \cdot \mathrm{K}^{-1} \cdot \mathrm{mol}^{-1}\right)$, and $\Delta_{\text {fus }} C p_{i}$ the difference between the heat capacity of liquid and solid phases; $\Delta_{\text {fus }} H_{i}$ and $T_{\mathrm{m}, i}$ are the melting enthalpy and temperature of the solute, respectively.

This equation assumes that the solid phases of each compound are immiscible and crystallize independently $\left(x_{i}^{\mathrm{s}} \gamma_{i}^{\mathrm{s}}\right.$ $=1$ ) as expected from a eutectic-type phase diagram. Moreover, in general the last term has a negligible value when compared with the first ${ }^{46,47}$ and thus was not considered in this work. The melting temperatures and enthalpies used in this work are reported in Table 1.

\section{RESULTS}

The solid-liquid phase diagrams measured in this work for the systems $[\mathrm{Ch}] \mathrm{Cl}+$ fatty acids and $[\mathrm{Ch}] \mathrm{Cl}+$ fatty alcohols are depicted in Figures 2 and 3, respectively. All the systems exhibited a phase behavior characterized by a single eutectic point at a temperature much lower than the melting temperature of pure $[\mathrm{Ch}] \mathrm{Cl}$, but surprisingly close to the melting temperature of the nonionic compound. The detailed experimental data are reported in the Supporting Information (Tables S1 and S2) for completeness. Data consistency was checked using tests proposed by Cunico et al. ${ }^{16}$ and Kang et al. $^{15}$ (detailed in the Supporting Information), and the results obtained are reported in Table S3. Values of the parameter $Q_{1}$ 

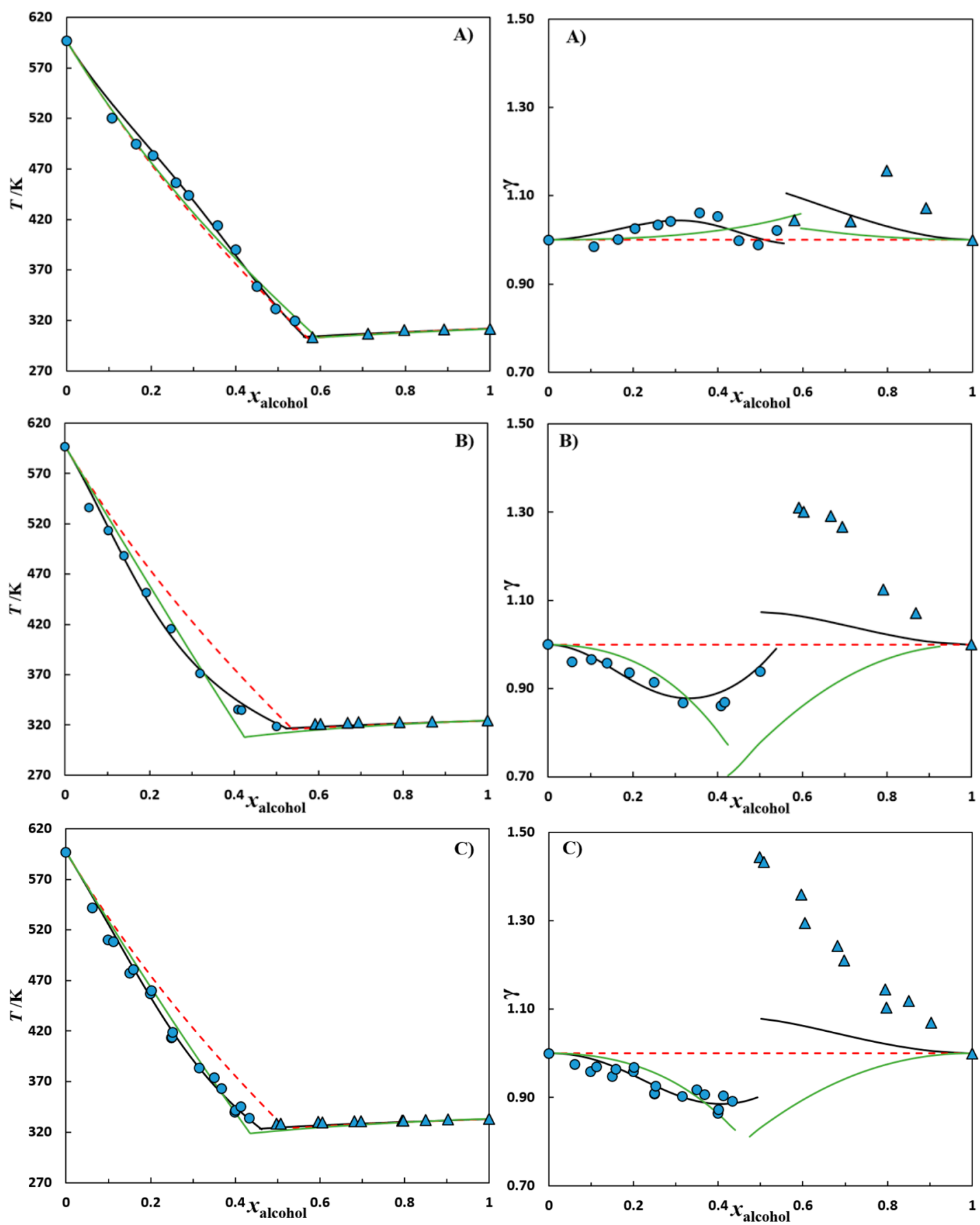

Figure 3. Solid-liquid phase diagrams for the binary mixtures composed of [Ch]Cl and (A) 1-tetradecanol, (B) 1-hexadecanol, and (C) 1octadecanol. $\boldsymbol{\Delta}$ and - represent the fatty alcohol and $[\mathrm{Ch}] \mathrm{Cl}$ experimental solubility curves measured in this work, while the lines depict the modeling results, namely, red dashed line, ideal; solid black line, PC-SAFT; and solid green line, NRTL.

(Kang et al. ${ }^{15}$ ) are greater than 0.5 for all systems, indicating that the quality of all data series passes this consistency. The pure component parameter, $Q_{2}$, of the consistency test proposed by Cunico et al. ${ }^{16}$ shows low values $(<0.1)$ for all the systems. However, these results suppose temperature differences, $\Delta t_{1}+\Delta t_{2} \leq 0.02 \mathrm{~K}$, which are, in fact, acceptable errors. The value of the global consistency parameter, $Q_{3}$ (Cunico et al. $^{16}$ ) is $>0.3$ for all the data series, so the values of the normalized root squared mean errors (nRSME) are always $<2 \%$, which is acceptable. The system capric acid $+[\mathrm{Ch}]$ $\mathrm{Cl}$ presents the lowest quality factors, while the binary palmitic acid $+[\mathrm{Ch}] \mathrm{Cl}$ shows the highest. These results of the two consistency analysis validate the experimental data presented in this work. The experimental activity coefficients of each compound in the liquid phase were estimated from the solidus curves using eq 16, and the resulting values are also depicted in Figures 2 and 3 and numerically reported in the Supporting Information.

The results obtained show that the binary systems composed of $[\mathrm{Ch}] \mathrm{Cl}$ and fatty acids, although commonly reported as examples of type III DESs, exhibit an almost ideal behavior, showing only slight positive deviations from the ideal behavior more evident in the fatty acid solubility curve, as depicted in Figure 2. The absence of negative deviations to ideality is 
strong evidence that the hydroxyl group present in $[\mathrm{Ch}] \mathrm{Cl}$ significantly influences the system behavior, because the huge negative deviations observed in the salt solubility curve for DESs composed of other quaternary ammonium salts and carboxylic acids reported by Pontes et al. $^{7}$ are no longer observed.

The systems with fatty alcohols show a slightly different behavior (probably because of a higher mobility of fatty alcohols when compared to the more rigid fatty acids) with positive deviations from the ideal behavior observed for the nonionic compound solubility curve, but exhibiting negative deviations from the ideal behavior in the $[\mathrm{Ch}] \mathrm{Cl}$ solubility curve for the systems with higher chain length alcohols (1hexadecanol and 1-octadecanol), suggesting the existence of favorable interactions between the hydroxyl group and the salt anion. Part of the negative deviation must also result from entropic effects due to the large size differences between the two compounds.

There are several advantages of modeling the measured phase diagrams, not only to achieve an accurate description of the experimental data (and reliable estimates of the eutectic points) but also to find information that can be gauged from the modeling results on the interactions between the mixtures components from the activity coefficients of these systems. Therefore, the eight phase diagrams here reported were modeled with eq 16 using three different methods: (i) considering an ideal liquid phase $\left(\gamma_{i}^{1}=1\right)$, or considering the nonideality of the liquid phase using activity coefficients calculated either with (ii) a $\mathrm{g}^{\mathrm{E}}$ model (NRTL) or (iii) an advanced association EoS (PC-SAFT). The SLE modeling results for the three methods are illustrated in Figures 2 and 3 along with the experimental data, and the accuracy of the different approaches can be evaluated through the deviations between the calculated melting temperatures, $T^{\text {calc }}$, and the experimental values, $T^{\exp }$. In this work, these deviations (depicted in Figure 4) are reported in terms of average

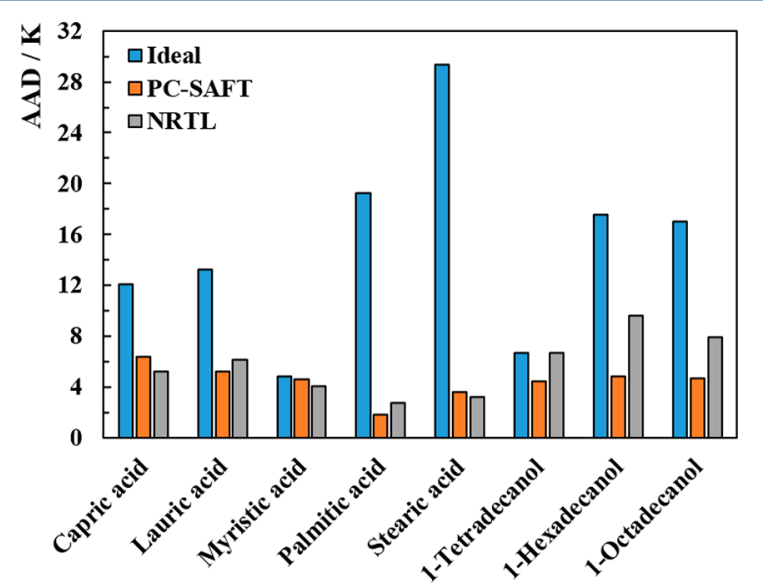

Figure 4. Average absolute deviations (K) for the three modeling approaches applied in this work.

absolute deviation (AAD) expressed as eq 17, where $N_{\text {exp }}$ is the total number of experimental data points for each system.

$$
\operatorname{AAD}(\mathrm{K})=\frac{1}{N_{\exp }} \sum_{k=1}^{N_{\text {exp }}} \mid \mathrm{T}_{k}^{\text {calc }}(\mathrm{K})-\mathrm{T}_{k}^{\exp }(\mathrm{K}) \mathrm{|}
$$

Figures 2 and 3 show that both PC-SAFT and NRTL are able to provide good descriptions of the SLE phase diagrams resulting in a considerable decrease of $\mathrm{AAD}$ if compared to the ideal solubility curves, which neglect activity coefficients in eq 16.

As described in section 3.3, NRTL was applied in its simplest form (without temperature dependence), using the parameters presented in Table 3. As shown in Table 3, the NRTL form

Table 3. Parameters of the NRTL Equation and Average Absolute Deviation for Each System Studied in This Work

\begin{tabular}{lcccc}
\multicolumn{1}{c}{ system } & \multicolumn{1}{c}{} & \multicolumn{1}{c}{ AAD } \\
1-tetradecanol $+[\mathrm{Ch}] \mathrm{Cl}$ & 0.20 & 64.7 & 347.4 & 6.7 \\
1-hexadecanol $+[\mathrm{Ch}] \mathrm{Cl}$ & 0.20 & -3287.6 & 653.1 & 9.6 \\
1-octadecanol $+[\mathrm{Ch}] \mathrm{Cl}$ & 0.20 & -2630.2 & 662.0 & 7.9 \\
capric acid $+[\mathrm{Ch}] \mathrm{Cl}$ & 0.20 & 13571.8 & -3457.2 & 5.2 \\
lauric acid $+[\mathrm{Ch}] \mathrm{Cl}$ & 0.20 & -4217.0 & 7073.1 & 6.1 \\
myristic acid $+[\mathrm{Ch}] \mathrm{Cl}$ & 0.20 & -943.3 & 795.1 & 4.0 \\
palmitic acid $+[\mathrm{Ch}] \mathrm{Cl}$ & 0.20 & -5102.6 & 9527.1 & 2.7 \\
stearic acid $+[\mathrm{Ch}] \mathrm{Cl}$ & 0.20 & -4883.8 & 9995.9 & 3.2 \\
\hline
\end{tabular}

applied uses three parameters, i.e., two interaction parameters $\left(a_{12}\right.$ and $\left.a_{21}\right)$ and the so-called nonrandomness parameter $\alpha_{12}$ that, in spite of having a clear physical meaning is often treated as an additional adjustable parameter. ${ }^{48}$ Renon and Prausnitz ${ }^{19}$ proposed some general rules depending on the compounds' family for the $\alpha_{12}$ value within the range $0.2-0.47$. In this work, in order to decrease the number of adjustable parameters within NRTL, a fixed value of $\alpha_{12}=0.2$ was found to provide good results for all the systems studied without any systematic loss of accuracy. Nevertheless, for the systems exhibiting negative deviations to the ideal behavior in the $[\mathrm{Ch}] \mathrm{Cl}$ solubility curve (e.g., $[\mathrm{Ch}] \mathrm{Cl}+1$-hexadecanol and $[\mathrm{Ch}] \mathrm{Cl}+$ 1-octadecanol), NRTL provides a considerably less accurate description of the experimental data regardless of the $\alpha_{12}$ applied. This is mainly due to the fact that NRTL does not account explicitly for the hydrogen-bonding interactions present between the two components.

Because of the physical meaning of its parameters, PC-SAFT provides a more convenient and usually better description of nonideal systems than NRTL, while providing additional insights into the systems' phase behavior and interactions. In order to obtain a quantitative description of the experimental data, binary parameters were applied, which are listed in Table 4. These binary parameters were estimated through the minimization of the $\operatorname{AAD}(T)$ in eq 17. By using the purecomponent parameters listed in Table 2 and only one binary

Table 4. PC-SAFT Binary Interaction Parameters and Average Absolute Deviation for Each System Studied in This Work

\begin{tabular}{|cccc} 
system & $k_{\text {ij_a }}$ & $k_{\text {ij_T }}$ & AAD (K) \\
\hline 1-tetradecanol $+[\mathrm{Ch}] \mathrm{Cl}$ & 0.0135 & 0.00021 & 4.4 \\
1-hexadecanol $+[\mathrm{Ch}] \mathrm{Cl}$ & 0.0135 & 0.00006 & 4.8 \\
1-octadecanol $+[\mathrm{Ch}] \mathrm{Cl}$ & 0.0135 & 0.00016 & 4.7 \\
capric acid $+[\mathrm{Ch}] \mathrm{Cl}$ & -0.0025 & 0.00010 & 6.4 \\
lauric acid $+[\mathrm{Ch}] \mathrm{Cl}$ & 0.0025 & 0.00012 & 5.2 \\
myristic acid $+[\mathrm{Ch}] \mathrm{Cl}$ & 0.0040 & 0.00012 & 2.8 \\
palmitic acid $+[\mathrm{Ch}] \mathrm{Cl}$ & 0.0110 & 0.00018 & 1.8 \\
stearic acid $+[\mathrm{Ch}] \mathrm{Cl}$ & 0.0170 & 0.00020 & 3.6
\end{tabular}



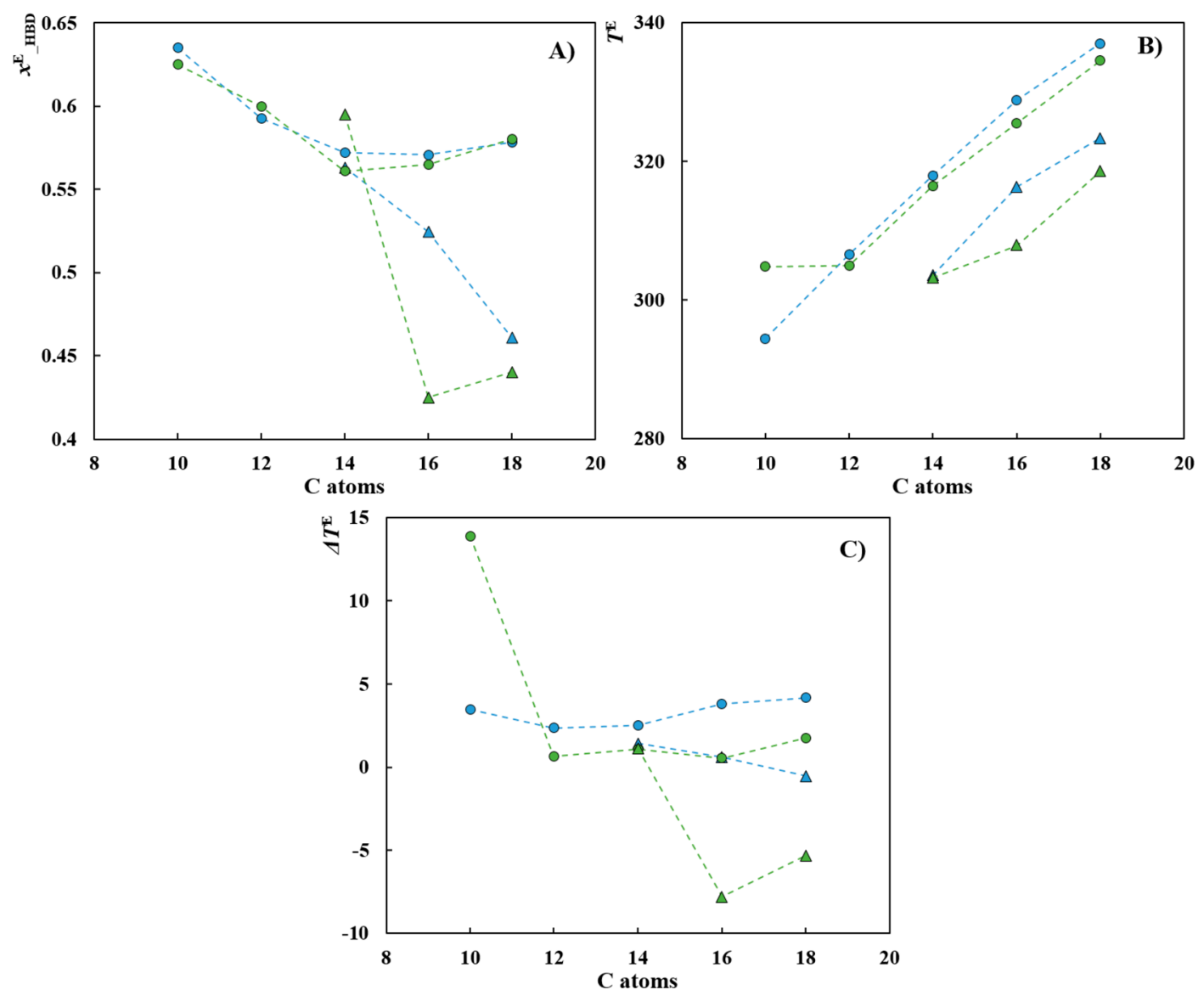

Figure 5. (A) Eutectic compositions (in mole fraction of nonionic compound), (B) eutectic temperatures, and (C) eutectic temperature difference to the ideal estimates for the different systems studied. The blue $\boldsymbol{\Delta}$ and $\boldsymbol{Q}$ stand for eutectic points predicted by PC-SAFT for systems containing fatty alcohols and fatty acids, respectively, while the green $\mathbf{\Delta}$ and $\boldsymbol{O}$ are the eutectic points predicted by NRTL for systems containing fatty alcohols and fatty acids, respectively. The dashed lines are guides for the eyes.

interaction parameter, PC-SAFT is able to accurately describe the phase diagrams of all the studied systems. As depicted in Figure 4, for most systems PC-SAFT allows a better description than NRTL, especially for mixtures $[\mathrm{Ch}] \mathrm{Cl}+$ fatty alcohols, in which negative deviations from ideal behavior were observed. This improved performance of PC-SAFT over NRTL is mainly due to the fact that PC-SAFT is able to capture complex nonideal behavior, such as activity coefficients greater than 1 for one component and lower than 1 for the other component (cf. Figure 3C). Furthermore, the acid/alcohol solubility curves only marginally depend on temperature, while the solubility curve of $[\mathrm{Ch}] \mathrm{Cl}$ is strongly temperature-dependent. This high accuracy of PC-SAFT is particularly remarkable because the $[\mathrm{Ch}] \mathrm{Cl}$ pure-component PC-SAFT parameters were regressed from experimental data obtained for very dilute aqueous solutions near room temperature. ${ }^{31}$ These parameters were used in this work to describe the SLE of water-free systems in a wide temperature and concentration ranges, in which interactions are very different compared to those observed in dilute aqueous solutions. However, as a consequence of the procedure adopted to obtain the $[\mathrm{Ch}] \mathrm{Cl}$ parameters, some of the physical features of the salt are not perfectly captured by the parametrization resulting in negative deviations from the ideal behavior being systematically predicted by PC-SAFT in the $[\mathrm{Ch}] \mathrm{Cl}$ solubility curve while predicting the ideal behavior experimentally observed for the HBD solubility curve. The results of such predictions $\left(k_{\mathrm{ij}}=0\right)$ are depicted in Figures S1 and S2 along with the accurate PC-SAFT correlations.

As both PC-SAFT and NRTL were able to provide a good description of the phase diagrams measured in this work, they can further be used to provide estimates of the eutectic points through the interception of the two solubility curves. The estimated eutectic points [both in composition $\left(x^{\mathrm{E}}\right)$ and in temperature $\left.\left(T^{\mathrm{E}}\right)\right]$ and the melting temperature difference compared to the ideal behavior $\left(\Delta T^{\mathrm{E}}=T_{\text {ideal }}^{\mathrm{E}}-T_{\text {real }}^{\mathrm{E}}\right)$ are depicted in Figure 5. As can be seen in Figure 5A, PC-SAFT and NRTL predict similar eutectic compositions for the systems $[\mathrm{Ch}] \mathrm{Cl}+$ fatty acids, while the predictions are much different for the systems $[\mathrm{Ch}] \mathrm{Cl}+$ fatty alcohols. Clearly, PCSAFT provides a smoother dependency on the eutectic composition for increasing chain lengths as one could expect from the near-ideal mixture behavior of these systems (all activity coefficients close to 1 ). In Figure 5B the predicted eutectic temperatures show a qualitative agreement between the two models with PC-SAFT displaying smoother linear trends for each family (specially for fatty acids). The linear increases on the eutectic temperatures are expected because they are mainly affected by the melting temperatures of the pure nonionic compounds, which usually follow a smooth increase with increasing chain length. As a consequence, PCSAFT predicts almost constant positive and small temperature differences when compared to the ideal behavior of each family (Figure $5 \mathrm{C}$ ), while in contrast, NRTL predicts a much wider 
range of temperature differences for the studied systems because of the clear overprediction and underestimation of the eutectic points for the systems capric acid $+[\mathrm{Ch}] \mathrm{Cl}$ (Figure 2A) and 1-hexadecanol $+[\mathrm{Ch}] \mathrm{Cl}$ (Figure 3B), respectively. The small temperature differences presented in Figure $5 \mathrm{C}$ challenge the current assumption that DESs, because they are dominated by hydrogen-bond interactions between the two components, are highly nonideal systems, and that this would be the reason for the freezing temperature depression observed on these systems. This suggests that systems that have been labeled as DESs, without a careful analysis of the liquid phase nonideality, are indeed just common eutectic mixtures.

Molecular based approaches such as PC-SAFT can provide insights into the systems' phase behavior and interactions because of the physical meaning of its parameters. The purecomponent parameters often correlate with the molecular weight within a homologous series providing a way to predict thermo-physical properties and phase behavior for compounds for which experimental data is not available. In a similar manner, the binary interaction parameters applied to describe mixture data often provide useful information regarding the systems' interactions. Therefore, for the systems $[\mathrm{Ch}] \mathrm{Cl}+$ fatty acids, both $k_{i j{ }_{1} \mathrm{a}}$ and $k_{i j \_}$were found to be positive and correlate linearly with the molecular weight of the carboxylic acid according to the following expressions:

$$
k_{i j \_a}=0.0024 M_{w_{-} \text {acid }}(\mathrm{g} / \mathrm{mol})-0.0268 ; R^{2}=0.9654
$$

$$
\begin{aligned}
k_{i j_{-} \mathrm{T}} & =1.300 \times 10^{-5} M_{\mathrm{w}_{-} \text {acid }}(\mathrm{g} / \mathrm{mol})-3.800 \times 10^{-5} ; \\
R^{2} & =0.8989
\end{aligned}
$$

The positive values observed for $k_{i j}$ in the $[\mathrm{Ch}] \mathrm{Cl}+$ fatty acids systems denote the systematic overestimation of the cross-dispersive interactions between $[\mathrm{Ch}] \mathrm{Cl}$ and the fatty acid. This is in agreement with the positive deviations from the ideal behavior observed from the experimental activity coefficients shown in Figure 2. Moreover, and because of the very limited number of systems containing fatty alcohols, a fixed value of $k_{i j \_\mathrm{a}}=0.0135$ was applied in this work, and positive values were also obtained for $k_{i j_{-} T}$. Thus, the same explanations apply to both $[\mathrm{Ch}] \mathrm{Cl}+$ fatty acids and $[\mathrm{Ch}] \mathrm{Cl}+$ fatty alcohol systems.

This kind of semipredictive modeling approach was also reported in our previous work ${ }^{7}$ on the modeling of SLE of DESs composed of symmetrical tetraalkylammonium chlorides and fatty acids. That work suggested the possible screening of SLE phase diagrams for eutectic mixtures by correlating binary parameters with chain length of the acids without the need of additional experimental data. In our previous work, a binary interaction parameter $\left(k_{i j \_ \text {eps }}\right)$ correcting the underestimation of the mixtures' cross-association energy given by the Wolbach and Sandler mixing rules ${ }^{32}$ was required to accurately describe the experimental phase diagrams. The values obtained for this parameter were found to increase (become more negative) with the increase of the alkyl chains, either of the alkyl chains within the ammonium salt or of those from fatty acids, suggesting a strengthening of the hydrogen-bonding interactions and thus on the negative deviations from ideality that ultimately were responsible for the huge melting temperature depressions observed experimentally leading to the formation of DESs. However, in this work such a parameter was not required to successfully describe the phase diagrams denoting the inexistence of stronger hydrogen bonding interactions in the mixture than those observed in the pure components.

Thus, it is clear that taking into account both the systems with $[\mathrm{Ch}] \mathrm{Cl}$ studied in this work and those with symmetrical tetra alkyl ammonium chlorides reported by Pontes et al., there is a decrease of the nonideality of the liquid phase of mixtures of fatty acids with quaternary ammonium salts with the decrease of the salts alkyl chain length and/or with the introduction of an hydroxyl group in the IL cation, following the trend

$$
\left[N_{3333}\right] \mathrm{Cl}>\left[N_{2222}\right] \mathrm{Cl}>\left[N_{1111}\right] \mathrm{Cl}>[\mathrm{Ch}] \mathrm{Cl}
$$

This trend is confirmed both experimentally (through the experimental values of the activity coefficients and the melting temperature differences observed) but also through the binary interaction parameter $\left(k_{i j \_ \text {eps }}\right)$ applied within PC-SAFT that systematically decreases the cross-association energy of the systems in that same order:

$$
-k_{i j \_ \text {eps }}\left(\left[N_{3333}\right] \mathrm{Cl}>\left[N_{2222}\right] \mathrm{Cl}>\left[N_{1111}\right] \mathrm{Cl}=0=[\mathrm{Ch}] \mathrm{Cl}\right)
$$

Hence, the question arising is why does the introduction of an hydroxyl group in the IL structure, which could be thought to allow for the formation of additional and stronger interactions with the HBD, have the opposite effect, leading to more ideal liquid mixtures. First, as already mentioned and previously discussed by Ashworth et al., ${ }^{49}$ all the possible $[\mathrm{Ch}] \mathrm{Cl}$ structures exhibit multiple hydrogen-bonding interactions, and the lowest-energy conformers feature a strong $\mathrm{OH} \cdots \mathrm{Cl}$ interaction (where the $\mathrm{OH}$ is only mildly affected by the charged ammonium center) while the weaker intramolecular $\mathrm{OH}-\mathrm{CH}$ hydrogen bonds are also preserved. The presence of these strong hydrogen bonding interactions in the pure IL, and the strong hydrogen bonding interactions also present in pure fatty acids and fatty alcohols, ${ }^{50}$ makes the difference between the interactions present in the pure compounds and those observed for the mixture negligible resulting in the quasi ideal behavior of the liquid phase and consequently in the absence of a significant melting temperature depression, particularly visible in the HBD melting curves.

\section{CONCLUSIONS}

The SLE phase diagrams for eight eutectic mixtures composed of $[\mathrm{Ch}] \mathrm{Cl}+$ fatty acids and $[\mathrm{Ch}] \mathrm{Cl}+$ fatty alcohols were measured experimentally using a visual detection technique. The consistency of the data obtained was evaluated and guaranteed using two consistency tests. The systems studied showed a quasi-ideal behavior displaying a eutectic temperature very close to the eutectic temperature predicted assuming an ideal liquid phase. These results, when compared to those obtained studying eutectic mixtures of symmetrical tetraalkylammonium chlorides with fatty acids, suggest that the presence of a hydroxyl group in the choline cation hinders the formation of stronger interactions with the hydrogen bond donor than those observed in the pure cholinium chloride, leading to near ideal liquid mixtures. Therefore, although commonly suggested as possible HBDs for [Ch]Cl-based DESs, monocarboxylic fatty acids and fatty alcohols are not able to form hydrogen-bond interactions with $[\mathrm{Ch}] \mathrm{Cl}$ strong enough to create large melting point depressions. These results show that mixtures that have been labeled as type III DESs based on cholinium chloride and mono carboxylic acids or alcohols, without a careful analysis of the liquid phase nonideality, are indeed just common eutectic mixtures. 
The phase diagrams were modeled by using the NRTL activity coefficient model and the molecular based PC-SAFT EoS. Both models were able to provide very good descriptions of the experimental data allowing for a significant decrease of the AAD when compared to the ideal solubility curves. Within NRTL, through the estimation of only two temperatureindependent interaction parameters, a good description was obtained for most of the systems. However, when negative deviations from the ideal behavior were observed, the NRTL performance deteriorates. Contrarily, PC-SAFT, using only one temperature-dependent binary interaction parameter (that was correlated with the molecular weight of the HBD), is able to provide a very good description of the phase diagrams measured in this work and is able to capture the versatile behavior of activity coefficients of all species. Moreover, as both models provided a good description of the SLE phase diagrams, they were used to estimate the eutectic points (both in composition and temperature), and PC-SAFT was shown to provide smoother trends for the eutectic points than NRTL. The physical meaning of the PC-SAFT binary parameters was also highlighted, and insights into the systems' behavior and interactions were given.

\section{ASSOCIATED CONTENT}

\section{S Supporting Information}

The Supporting Information is available free of charge on the ACS Publications website at DOI: 10.1021/acs.iecr.7b02382.

Experimental data, consistency tests, and PC-SAFT results (PDF)

\section{AUTHOR INFORMATION}

\section{Corresponding Author}

*E-mail: jcoutinho@ua.pt. Phone: +351 234370200. Fax: +351 234370084.

\section{ORCID $\odot$}

Emanuel A. Crespo: 0000-0003-2137-0564

Mónia A. R. Martins: 0000-0003-0748-1612

Gabrielle Sadowski: 0000-0002-5038-9152

Christoph Held: 0000-0003-1074-177X

Simão P. Pinho: 0000-0002-9211-857X

João A. P. Coutinho: 0000-0002-3841-743X

\section{Notes}

The authors declare no competing financial interest.

\section{ACKNOWLEDGMENTS}

This work was developed in the scope of Projects POCI-010145-FEDER-007679 - CICECO - Aveiro Institute of Materials (ref. FCT UID/CTM/50011/2013), POCI-01-0145-FEDER006984 - Associate Laboratory LSRE-LCM both funded by European Regional Development Fund (ERDF) through COMPETE2020 - Programa Operacional Competitividade e Internacionalização (POCI), and by national funds through FCT - Fundação para a Ciência e a Tecnologia. This work is also a result of project “AIProcMat@N2020 - Advanced Industrial Processes and Materials for a Sustainable Northern Region of Portugal 2020", with the reference NORTE-01-0145FEDER-000006, supported by Norte Portugal Regional Operational Programme (NORTE 2020), under the Portugal 2020 Partnership Agreement, through ERDF. M.A.R.M acknowledges FCT for her Ph.D. grant (SFRH/BD/87084/
2012). C.H. gratefully acknowledges financial support of MaxBuchner Research Foundation.

\section{REFERENCES}

(1) Smith, E. L.; Abbott, A. P.; Ryder, K. S. Deep Eutectic Solvents (DESs) and Their Applications. Chem. Rev. 2014, 114, 11060-11082. (2) Dai, Y.; Spronsen, J.; Witkamp, G.-J.; Verpoorte, R.; Choi, Y. H. Natural Deep Eutectic Solvents as New Potential Media for Green Technology. Anal. Chim. Acta 2013, 766, 61.

(3) Abbott, A. P.; Capper, G.; Davies, D. L.; Rasheed, R. K.; Tambyrajah, V. Novel Solvent Properties of Choline Chloride/urea Mixtures. Chem. Commun. (Cambridge, U. K.) 2003, 70-71.

(4) Morrison, H. G.; Sun, C. C.; Neervannan, S. Characterization of Thermal Behavior of Deep Eutectic Solvents and Their Potential as Drug Solubilization Vehicles. Int. J. Pharm. 2009, 378, 136-139.

(5) Abbott, A. P.; Boothby, D.; Capper, G.; Davies, D. L.; Rasheed, R. K. Deep Eutectic Solvents Formed between Choline Chloride and Carboxylic Acids: Versatile Alternatives to Ionic Liquids. J. Am. Chem. Soc. 2004, 126, 9142-9147.

(6) Florindo, C.; Oliveira, F. S.; Rebelo, L. P. N.; Fernandes, A. M.; Marrucho, I. M. Insights into the Synthesis and Properties of Deep Eutectic Solvents Based on Choline Chloride and Carboxylic Acids. ACS Sustainable Chem. Eng. 2014, 2, 2416-2425.

(7) Pontes, P. V. A.; Crespo, E. A.; Martins, M. A. R.; Silva, L. P.; Neves, C. M. S. S.; Máximo, G. J.; Hubinger, M. D.; Batista, E. A. C.; Pinho, S. P.; Coutinho, J. A. P.; et al. Measurement and PC-SAFT Modeling of Solid-Liquid Equilibrium of Deep Eutectic Solvents of Quaternary Ammonium Chlorides and Carboxylic Acids. Fluid Phase Equilib. 2017, 448, 69.

(8) Shahbaz, K.; Mjalli, F. S.; Hashim, M. A.; Al-Nashef, I. M. Using Deep Eutectic Solvents for the Removal of Glycerol from Palm OilBased Biodiesel. J. Appl. Sci. 2010, 10, 3349-3354.

(9) Shahbaz, K.; Mjalli, F. S.; Hashim, Ma.; AlNashef, I. M. Prediction of Deep Eutectic Solvents Densities at Different Temperatures. Thermochim. Acta 2011, 515, 67-72.

(10) Shahbaz, K.; Baroutian, S.; Mjalli, F. S.; Hashim, M. A.; Alnashef, I. M. Densities of Ammonium and Phosphonium Based Deep Eutectic Solvents: Prediction Using Artificial Intelligence and Group Contribution Techniques. Thermochim. Acta 2012, 527, 59-66.

(11) Shahbaz, K.; Mjalli, F. S.; Hashim, M. A.; AlNashef, I. M. Prediction of the Surface Tension of Deep Eutectic Solvents. Fluid Phase Equilib. 2012, 319, 48-54.

(12) Choi, Y. H.; Van Spronsen, J.; Dai, Y.; Verberne, M.; Hollmann, F.; Arends, I. W. C. E.; Witkamp, G. J.; Verpoorte, R. Are Natural Deep Eutectic Solvents the Missing Link in Understanding Cellular Metabolism and Physiology? Plant Physiol. 2011, 156, 1701-1705.

(13) Maugeri, Z.; Dominguez de Maria, P. Novel Choline-ChlorideBased Deep-Eutectic-Solvents with Renewable Hydrogen Bond Donors: Levulinic Acid and Sugar-Based Polyols. RSC Adv. 2012, 2, 421-425.

(14) Dai, Y.; Van Spronsen, J.; Witkamp, G. J.; Verpoorte, R.; Choi, Y. H. Ionic Liquids and Deep Eutectic Solvents in Natural Products Research: Mixtures of Solids as Extraction Solvents. J. Nat. Prod. 2013, $76,2162-2173$.

(15) Kang, J. W.; Diky, V.; Chirico, R. D.; Magee, J. W.; Muzny, C. D.; Kazakov, A. F.; Kroenlein, K.; Frenkel, M. Algorithmic Framework for Quality Assessment of Phase Equilibrium Data. J. Chem. Eng. Data 2014, 59, 2283-2293.

(16) Cunico, L. P.; Ceriani, R.; Sarup, B.; O’Connell, J. P.; Gani, R. Data, Analysis and Modeling of Physical Properties for Process Design of Systems Involving Lipids. Fluid Phase Equilib. 2014, 362, 318-327. (17) Fernandez, L.; Silva, L. P.; Martins, M. A. R.; Ferreira, O.; Ortega, J.; Pinho, S. P.; Coutinho, J. A. P. Indirect Assessment of the Fusion Properties of Choline Chloride from Solid-Liquid Equilibria Data. Fluid Phase Equilib. 2017, 448, 9.

(18) Gross, J.; Sadowski, G. Perturbed-Chain SAFT: An Equation of State Based on a Perturbation Theory for Chain Molecules. Ind. Eng. Chem. Res. 2001, 40, 1244-1260. 
(19) Renon, H.; Prausnitz, J. M. Local Compositions in Thermodynamic Excess Functions for Liquid Mixtures. AIChE J. 1968, 14, 135-144.

(20) de Matos, F. C.; da Costa, M. C.; de Almeida Meirelles, A. J.; Batista, E. A. C. Binary Solid-liquid Equilibrium Systems Containing Fatty Acids, Fatty Alcohols and Triolein by Differential Scanning Calorimetry. Fluid Phase Equilib. 2015, 404, 1-8.

(21) de Matos, F. C.; da Costa, M. C.; Meirelles, A. J. d. A.; Batista, E. A. C. Binary Solid-liquid Equilibrium Systems Containing Fatty Acids, Fatty Alcohols and Trilaurin by Differential Scanning Calorimetry. Fluid Phase Equilib. 2016, 423, 74-83.

(22) Jackson, G.; Chapman, W. G.; Gubbins, K. E. Phase Equilibria of Associating Fluids of Spherical and Chain Molecules. Int. J. Thermophys. 1988, 9, 769-779.

(23) Chapman, W. G.; Jackson, G.; Gubbins, K. E. Phase Equilibria of Associating Fluids. Chain Molecules with Multiple Bonding Sites. Mol. Phys. 1988, 65, 1057-1079.

(24) Chapman, W. G.; Gubbins, K. E.; Jackson, G.; Radosz, M. SAFT: Equation-of-State Solution Model for Associating Fluids. Fluid Phase Equilib. 1989, 52, 31-38.

(25) Chapman, W. G.; Gubbins, K. E.; Jackson, G.; Radosz, M. New Reference Equation of State for Associating Liquids. Ind. Eng. Chem. Res. 1990, 29, 1709-1721.

(26) Wertheim, M. S. Fluids with Highly Directional Attractive Forces. I. Statistical Thermodynamics. J. Stat. Phys. 1984, 35, 19-34.

(27) Wertheim, M. S. Fluids with Highly Directional Attractive Forces. II. Thermodynamic Perturbation Theory and Integral Equations. J. Stat. Phys. 1984, 35, 35-47.

(28) Wertheim, M. S. Fluids with Highly Directional Attractive Forces. III. Multiple Attraction Sites. J. Stat. Phys. 1986, 42, 459-476.

(29) Wertheim, M. S. Fluids with Highly Directional Attractive Forces. IV. Equilibrium Polymerization. J. Stat. Phys. 1986, 42, 477492.

(30) Verevkin, S. P.; Sazonova, A. Y.; Frolkova, A. K.; Zaitsau, D. H.; Prikhodko, I. V.; Held, C. Separation Performance of BioRenewable Deep Eutectic Solvents. Ind. Eng. Chem. Res. 2015, 54, 3498-3504.

(31) Zubeir, L. F.; Held, C.; Sadowski, G.; Kroon, M. C. PC-SAFT Modeling of CO2 Solubilities in Deep Eutectic Solvents. J. Phys. Chem. B 2016, 120, 2300-2310.

(32) Wolbach, J. P.; Sandler, S. I. Using Molecular Orbital Calculations To Describe the Phase Behavior of Cross-Associating Mixtures. Ind. Eng. Chem. Res. 1998, 37, 2917-2928.

(33) Ji, X.; Held, C.; Sadowski, G. Modeling Imidazolium-Based Ionic Liquids with ePC-SAFT. Fluid Phase Equilib. 2012, 335, 64-73.

(34) Nann, A.; Mündges, J.; Held, C.; Verevkin, S. P.; Sadowski, G. Molecular Interactions in 1-Butanol + IL Solutions by Measuring and Modeling Activity Coefficients. J. Phys. Chem. B 2013, 117, 31733185.

(35) Passos, H.; Khan, I.; Mutelet, F.; Oliveira, M. B.; Carvalho, P. J.; Santos, L. M. N. B. F.; Held, C.; Sadowski, G.; Freire, M. G.; Coutinho, J. A. P. Vapor-Liquid Equilibria of Water + Alkylimidazolium-Based Ionic Liquids: Measurements and Perturbed-Chain Statistical Associating Fluid Theory Modeling. Ind. Eng. Chem. Res. 2014, 53, 3737-3748.

(36) Albers, K.; Heilig, M.; Sadowski, G. Reducing the Amount of PCP-SAFT Fitting Parameters. 2. Associating Components. Fluid Phase Equilib. 2012, 326, 31-44.

(37) Daubert, T. E.; Sibul, H. M.; Stebbins, C. C.; Danner, R. P.; Rowley, R. L.; Adams, M. E.; Wilding, W. V; Marshall, T. L. Physical and Thermodynamic Properties of Pure Chemicals: DIPPR: Data Compilation: Core + Supplements 1-10; Taylor \& Francis: Washington, DC, 2000.

(38) Albers, K.; Sadowski, G. Reducing the Amount of PCP-SAFT Fitting Parameters. 1. Non-Polar and Dipolar Components. Fluid Phase Equilib. 2012, 326, 21-30.

(39) Huang, S. H.; Radosz, M. Equation of State for Small, Large, Polydisperse and Associating Molecules. Ind. Eng. Chem. Res. 1990, 29, 2284-2294.
(40) Mulyono, S.; Hizaddin, H. F.; Alnashef, I. M.; Hashim, M. A.; Fakeeha, A. H.; Hadj-Kali, M. K. Separation of BTEX Aromatics from N-Octane Using a (Tetrabutylammonium Bromide + Sulfolane) Deep Eutectic Solvent - Experiments and COSMO-RS Prediction. RSC Adv. 2014, 4, 17597-17606.

(41) Hizaddin, H. F.; Sarwono, M.; Hashim, M. A.; Alnashef, I. M.; Hadj-Kali, M. K. Coupling the Capabilities of Different Complexing Agents into Deep Eutectic Solvents to Enhance the Separation of Aromatics from Aliphatics. J. Chem. Thermodyn. 2015, 84, 67-75.

(42) Hizaddin, H. F.; Hadj-Kali, M. K.; Ramalingam, A.; Ali Hashim, M. Extractive Denitrogenation of Diesel Fuel Using Ammonium- and Phosphonium-Based Deep Eutectic Solvents. J. Chem. Thermodyn. 2016, 95, 164-173.

(43) Kareem, M. A.; Mjalli, F. S.; Hashim, M. A.; Hadj-Kali, M. K. O.; Bagh, F. S. G.; Alnashef, I. M. Phase Equilibria of Toluene/heptane with Tetrabutylphosphonium Bromide Based Deep Eutectic Solvents for the Potential Use in the Separation of Aromatics from Naphtha. Fluid Phase Equilib. 2012, 333, 47-54.

(44) Rodriguez, N. R.; Molina, B. S.; Kroon, M. C. Aliphatic+ethanol Separation via Liquid-liquid Extraction Using Low Transition Temperature Mixtures as Extracting Agents. Fluid Phase Equilib. 2015, 394, 71-82.

(45) Sander, A.; Rogošić, M.; Slivar, A.; Žuteg, B. Separation of Hydrocarbons by Means of Liquid-Liquid Extraction with Deep Eutectic Solvents. Solvent Extr. Ion Exch. 2016, 34, 86-98.

(46) Gmehling, J.; Kolbe, B.; Kleiber, M.; Rarey, J. Chemical Thermodynamics for Process Simulation; Wiley-VCH: Weinheim, Germany, 2012.

(47) Coutinho, J. A. P.; Andersen, S. I.; Stenby, E. H. Evaluation of Activity Coefficient Models in Prediction of Alkane Solid-Liquid Equilibria. Fluid Phase Equilib. 1995, 103, 23-39.

(48) Kontogerogis, G. M.; Folas, G. K. Thermodynamic Models for Industrial Applications - from Classical and Advanced Mixing Rules to Association Theories; John Wiley \& Sons: Chichester, U.K., 2010.

(49) Ashworth, C. R.; Matthews, R. P.; Welton, T.; Hunt, P. A. Doubly Ionic Hydrogen Bond Interactions within the Choline Chloride-Urea Deep Eutectic Solvent. Phys. Chem. Chem. Phys. 2016, $18,18145-18160$.

(50) Kleiner, M.; Tumakaka, F.; Sadowski, G. Thermodynamic Modeling of Complex Systems. In Molecular Thermodynamics of Complex Systems; Lu, X., Hu, Y., Eds.; Springer: Berlin, 2009; pp 75108. 